\title{
CFD Analysis on Aerodynamic Coefficients of Flying Saucer
}

\author{
N Ruseno ${ }^{1, *}$ \\ 1 International University Liaison Indonesia, My Republic Plaza, $5^{\text {th }}$ Floor, BSD City, Tangerang, Banten \\ 15345, Indonesia \\ * Correspondence: neno.ruseno@iuli.ac.id
}

Received: 12 January 2021; Accepted: 10 May 2021; Published: 30 June 2021

With the growing of Unmanned Aerial Vehicle (UAV) usage, many new types of UAV are introduced. Flying Saucer is a new type of UAV which is not yet famous in the market. The aim of this study is to analysis the aerodynamic coefficients of a Flying Saucer. The research question arise is What the optimum angle of attack for Flying Saucer flight is. The study is conducted in Computational Fluid Dynamics (CFD) using COMSOL Multiphysics with Laminar Flow physics for several angles of attack. The analysis considers Lift and Drag coefficient in the form of $C_{L}$ and $C_{D}$ to angle of attack $(\alpha)$ plot, ratio of $C_{L} / C_{D}$ to angle of attack $(\alpha)$ plot and drag polar plot. We conclude that a symmetric Flying Sauce has aerodynamic characteristic with the optimum operational angle of attack in the range of 8 to $16 \mathrm{deg}$. The $C_{D}$ and $C_{L}$ has a quadratic relationship with large $C_{D 0}$ due to the geometric of Flying Saucer. It recommends that further study should explore in the area of zero and maximum angle of attack $(\alpha)$ and validation in wind tunnel experiment.

Keywords: Flying saucer, aerodynamic coefficients, angle of attack, CFD, COMSOL Multiphysics

\section{Introduction}

Unmanned Aerial Vehicle (UAV) grows significantly in recent years. It becomes part of our daily life in many aspects such as photography, survey, monitoring, search \& rescue, delivery, etc. The data from the US commercial drone market shows a linear grow from 2014 at around 300 million USD until 2020 at around 850 million USD and predicted to be continued [1].

There are many types of UAV currently available in the market such as multi-copter, fixed wing, e-VTOL, etc. The research on UAV is also growing, many types of UAV and its technology is developed to suit the market. One of the new UAV types is a Flying Saucer. It's geometry looks like a famous Unidentified Flying Object (UFO) [2]. So far, there are limited research published to study its aerodynamic characteristics.

In this study, the assumptions have been made on micro-size UAV, laminar flow, low speed, and neglect heat transfer phenomenon. This study used COMSOL Multiphysics as the application to simulate the Flying Saucer in computational fluid dynamic environment [3].

The aim of this study is to analysis the aerodynamic coefficients of a Flying Saucer. It reported with a literature review, background theory, methodology, results and analysis and then conclusions. The main research question for this study is on how to find the optimum angle of attack for Flying Saucer flight. 


\section{Literature Review}

There are very limited publications related with aerodynamic analysis of Flying Saucer. We found only 4 publications which all of them used Computational Fluid Dynamics (CFD) methods and we can categorize it based on the geometry used as Flying Saucer and Coanda Saucer.

In the Flying Saucer category, the two publications used similar geometry as shown in Figure 1 (a) as ellipsoid and (b) as ring airfoil. However, the assumed air flow direction is different. The first publication used horizontal air flow [4], but the second one use vertical air flow [5].

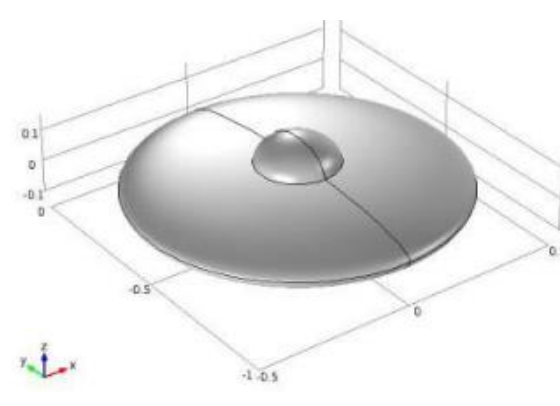

(a)

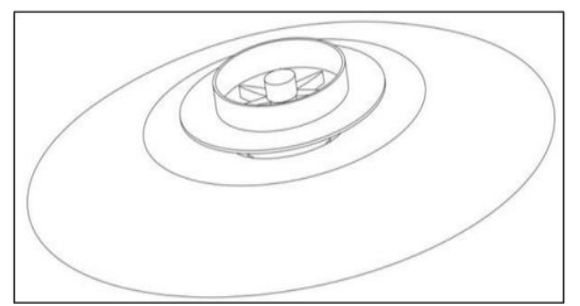

(b)
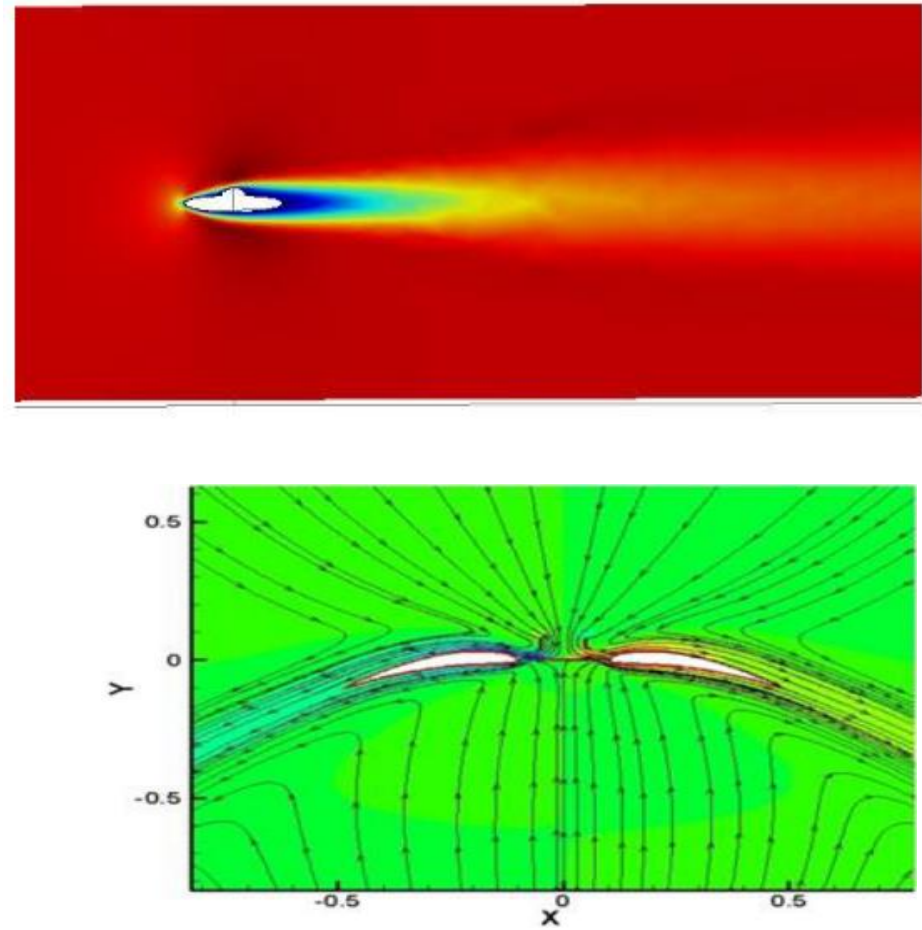

Figure 1. Flying Saucer geometry and air flow direction: (a) ellipsoid [4], (b) ring airfoil [5]

Both resulted aerodynamic characteristics are in the form of $C_{L}$ and $C_{D}$. The first publication resulted one simulation condition which provide $C_{D}=0.21934$ and $C_{L}=77.845 \times 10-7$ [4]. However, the second publication provide the $C_{L}$ and $C_{D}$ in the form of plot for several angles of attack $(\alpha)$ as shown in Figure 2 [5].

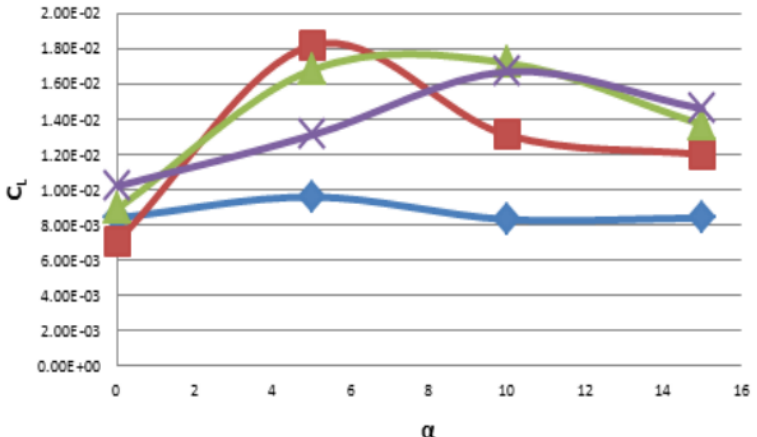

(a)

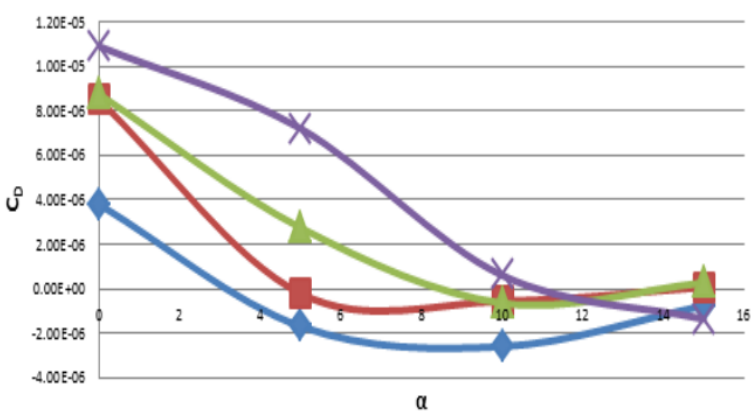

(b) 


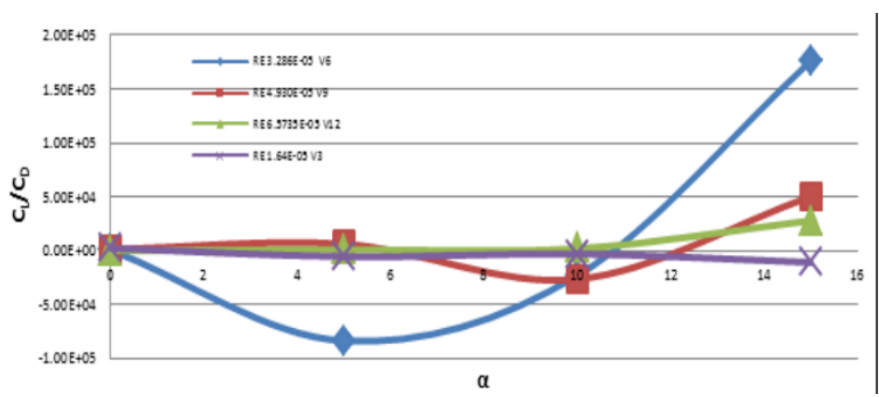

(c)

Figure 2. $C L$ and $C D$ values of Flying Saucer [5] : (a) $C_{L}$ vs alpha, (b) $C_{D}$ vs $\alpha$, (c) $C_{L} / C_{D}$ vs $\alpha$

For the second category which is the Coanda Saucer, there are 2 publications found. The basic geometries are the same, but the first one in this category used two propeller [6] and the second one only one propeller [7] as shown in Figure 3. Both analyses used CFD with vertical flow direction.
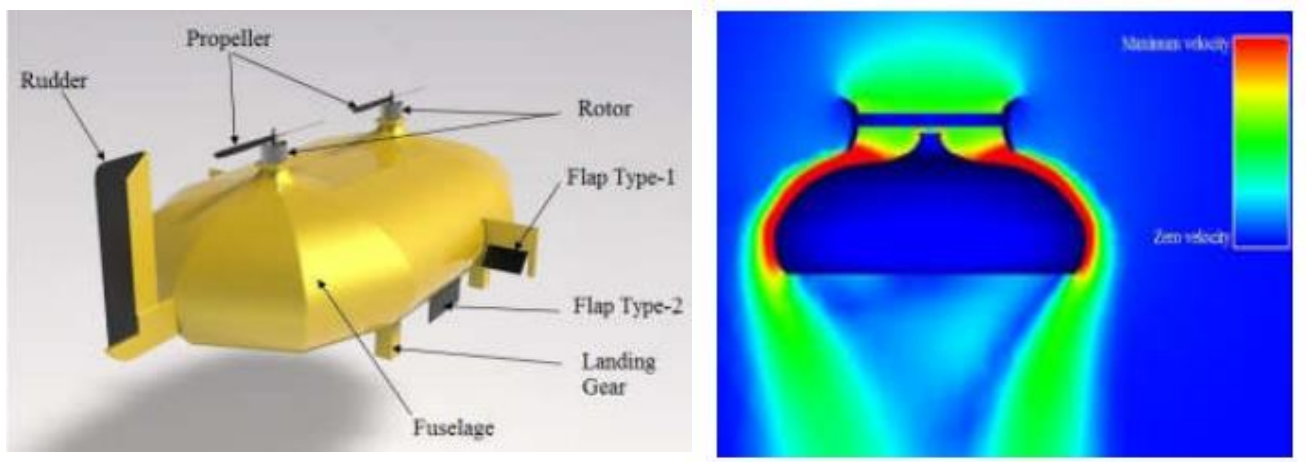

(a)
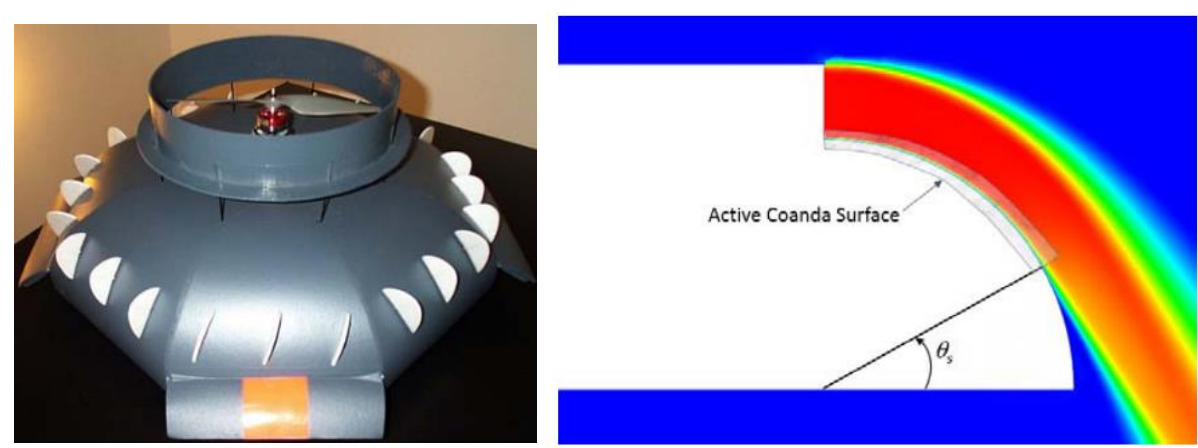

(b)

Figure 3. Coanda Saucer geometry and air flow: (a) two propellers [6], (b) one propeller [7]

The resulted parameter from both publication is Lift force as shown in Figure 4. The first publication visualized the lift force vector around the body [6]. However, the second publication shown it in the form of Lift force vs $\mathrm{h} / \mathrm{R}$ plot, which $\mathrm{h}$ is the height of the outlet flow and $\mathrm{R}$ is the radius of Coanda surface [7]. 


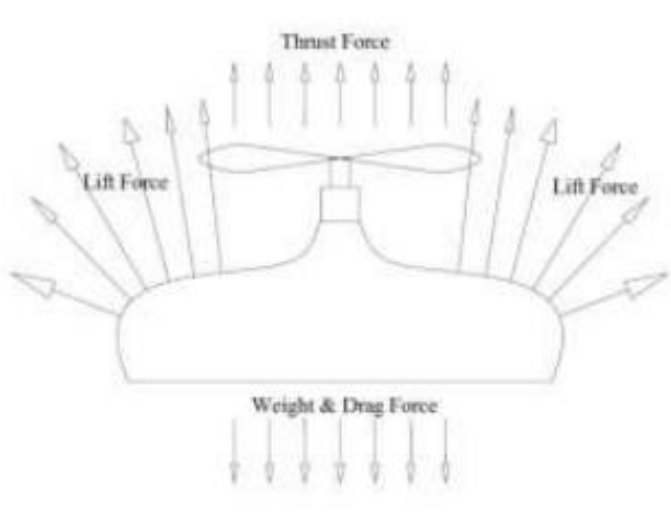

(a)

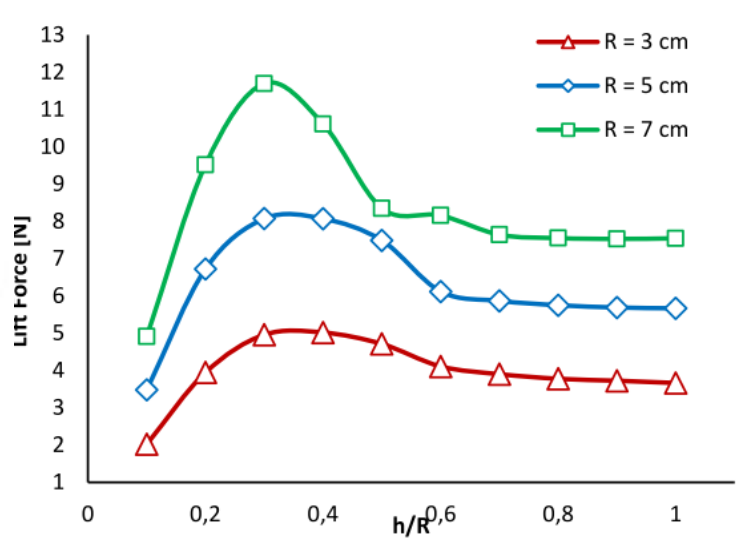

(b)

Figure 4. Lift Force of Coanda Saucer: (a) Lift vector [6], (b) Lift force vs h/R plot [7]

\section{Background theory}

The governing equation for computational fluid dynamics consist of two equations [4],[8]. The first one is mass conservation equation:

$$
\frac{\partial \rho}{\partial t}+\nabla \cdot(\rho \vec{v})=0
$$

and the second is Navier-Stokes equation:

$$
\rho\left(\frac{\partial \vec{v}}{\partial t}+\vec{v} \cdot \nabla \vec{v}\right)=-\nabla p+\nabla \cdot \vec{T}+\vec{F}
$$

$$
\text { where: } \begin{aligned}
& \rho=\text { density } \\
& \mathrm{v}=\text { velocity } \\
& \mathrm{t}=\text { time } \\
& \nabla=\text { gradient } \\
& \mathrm{p}=\text { pressure } \\
& \mathrm{T}=\text { stress } \\
& \mathrm{F}=\text { body forces }
\end{aligned}
$$

The mass conservation equation states that there must be no changes of mass in the moving airflow inside the closed system of our consideration. However, the Navier-Stokes equation gives a balance between acceleration of a small region of fluid equal to the forces acting on it.

The forces caused by the fluid motion around a body is Lift and Drag forces. The Lift is perpendicular to the airflow and the Drag is parallel to the airflow. We assume no side slip angle; thus, the Side force can be neglected. The equation to calculate the Lift force is shown in equation (3). The Drag force equation has the similar pattern but with Drag coefficient.

$$
L=\frac{1}{2} \rho v^{2} S C_{L}
$$

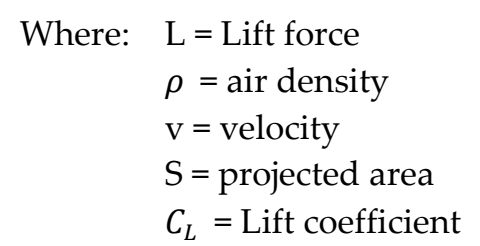




\section{Research Methods}

The research conducted a computational fluid dynamic method on a Flying Saucer. A laminar flow study in COMSOL Multiphysics application is used to analyze aerodynamic characteristic of the Flying Saucer.

The flow of process in this study shown in Figure 5. It starts with setup the geometry including the flying saucer and the fluid then setup simulation required for Laminar Flow physics. We run several simulations for several angle of attack $(\alpha)$ from 0 deg until $20 \mathrm{deg}$. Then, we retrieve the Lift and Drag Force using surface integration function. The collected data then processed to calculate the Lift and Drag Coefficient. Then the data is plotted and analyzed.

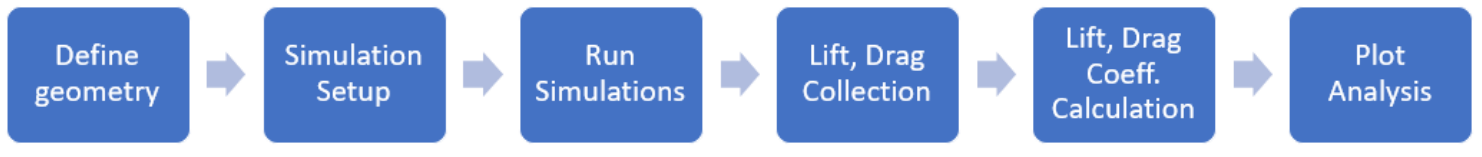

Figure 5. Flow Process of the study

The geometry of Flying Saucer used for this simulation made of an ellipsoid and a sphere with dimension as shown in Figure 6 (a) in mm. The geometry of air used as the medium of flying is in the form of a block with air properties are taken from Built in material available in the application.

In the Laminar Flow study, we need to define several boundaries such as Inlet, Outlet, Wall, and Symmetry. The Inlet velocity is $0.5 \mathrm{~m} / \mathrm{s}$ due to a low-speed assumption and the Outlet has 0 pressure.

The mesh created by user defined parameters with the size setup from Coarse at the outer wall and Finer in the inner wall. The resulted mesh is shown in Figure 6 (b). The mesh is calibrated for Fluid Dynamics. From statistics of mesh, it consists of 491,084 elements with minimum quality of 0.01878 and average quality of 0.6921 . Stationary study is selected to generate the result.

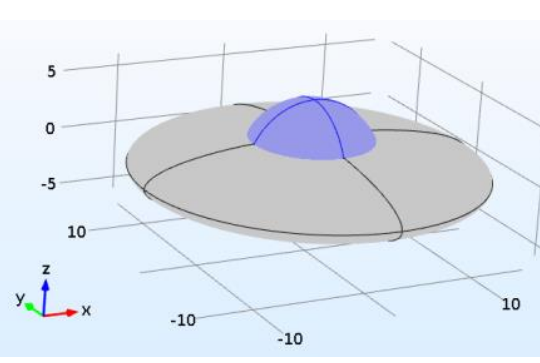

(a)

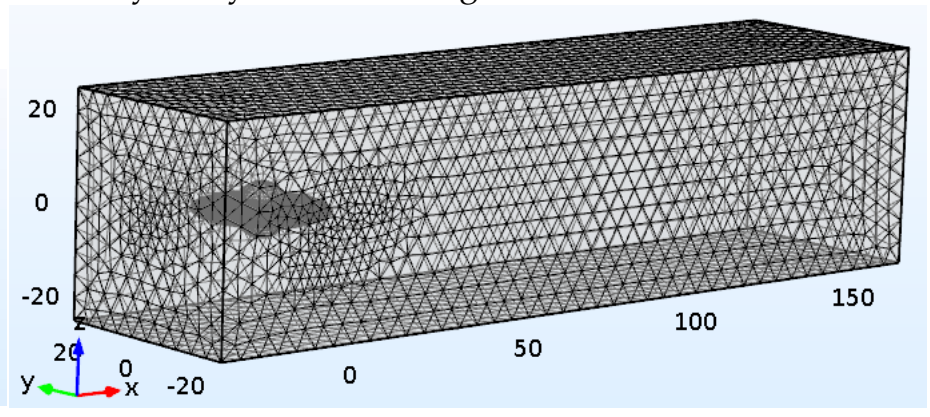

(b)

Figure 6. (a) The geometry of Flying Saucer, (b) The mesh generated

\section{Results and Discussions}

After the run of 9 simulation scenarios for the angle of attack $(\alpha)$ range between 0 to $20 \mathrm{deg}$. The first $6 \alpha(0,2.5,5,10,15,16 \mathrm{deg})$ successfully got convergence results. However, the last $3 \alpha(17,18$, $20 \mathrm{deg}$ ) got divergence results. Thus, we will present here the convergence results only.

The result of Laminar Flow study of Flying Saucer can be visualized in the Figure 7. It shows the air flow in the form of streamlines, the pressure on the surface of Flying Saucer is visualize in contour surface and the pressure on Symmetry boundary also shown in blue line. 


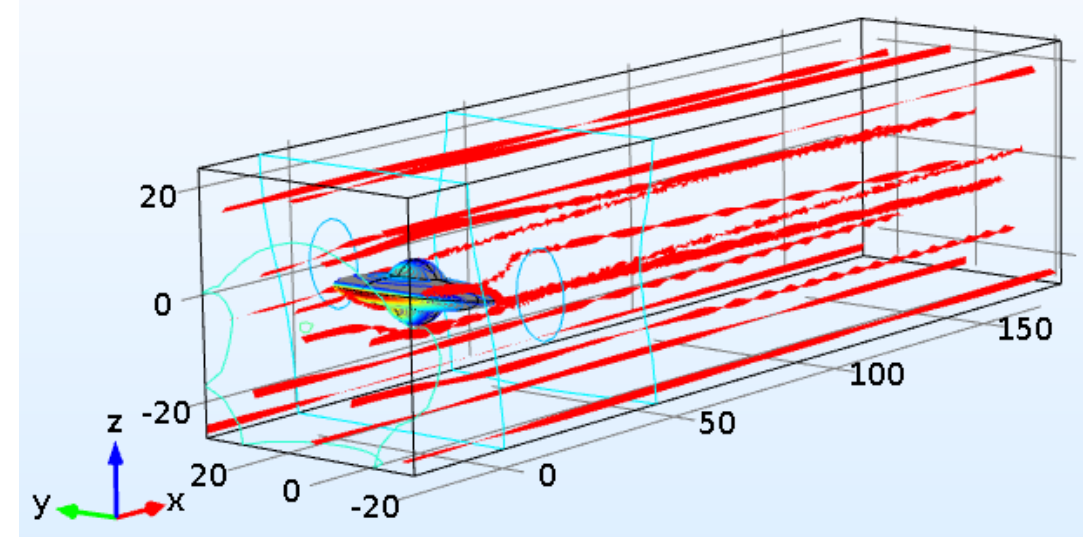

Figure 7. Visualization of Laminar Flow around Flying Saucer.

The velocity and pressure profile of Flying Saucer for each angle of attack $(\alpha)$ is shown in Figure 8 and 9. The velocity of flow near the object greatly affected by the position of the Flying Saucer relative to the air flow. The pressure on the lower side of Flying Saucer increases significantly with the increase of angle of attack $(\alpha)$.

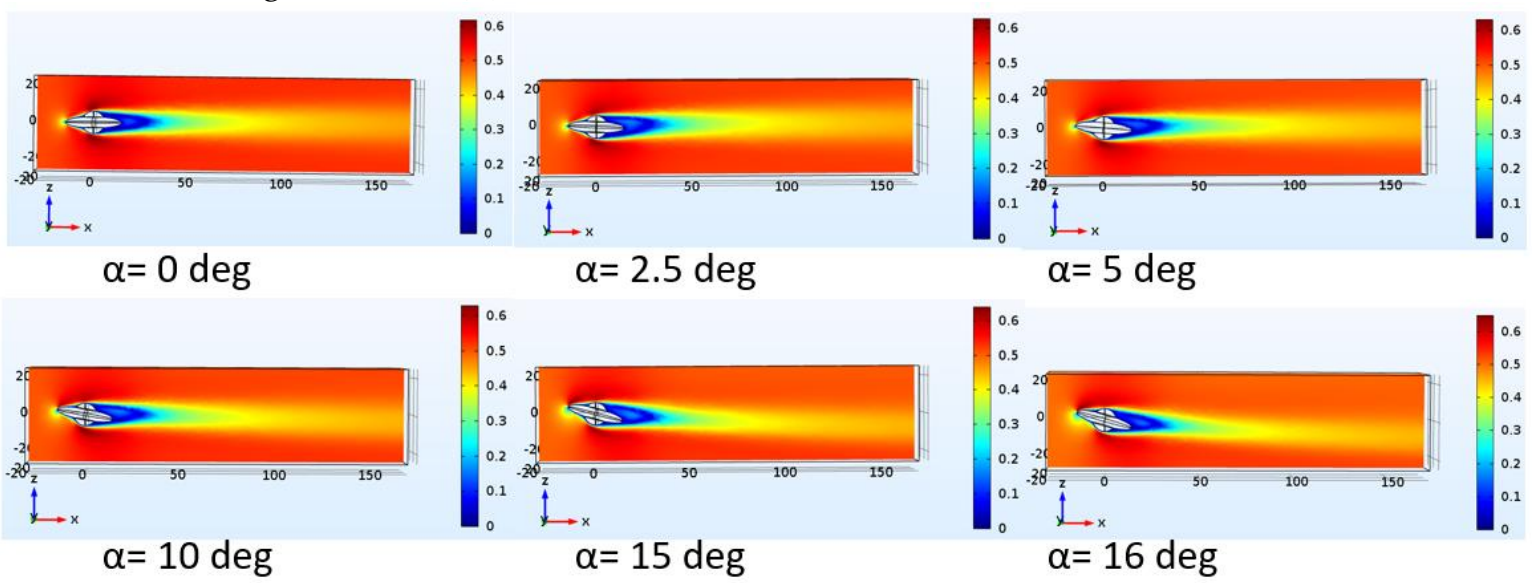

Figure 8. Velocity profile of Flying Saucer for several angle of attack $(\alpha)$.

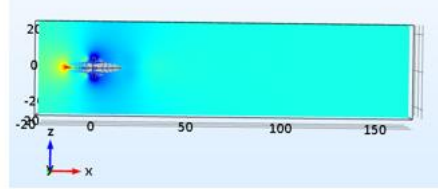

$\alpha=0$ deg

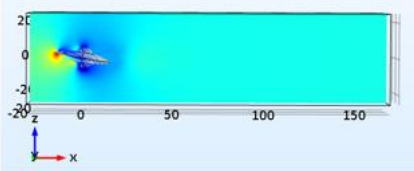

$\alpha=10 \mathrm{deg}$

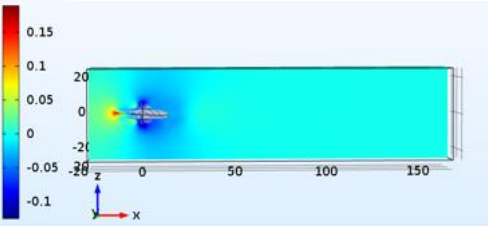

$\alpha=2.5 \mathrm{deg}$

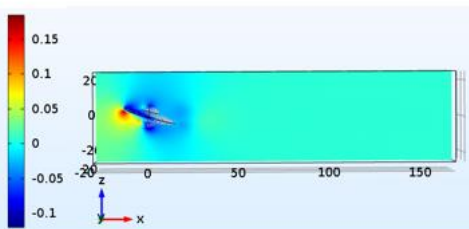

$\alpha=15 \mathrm{deg}$

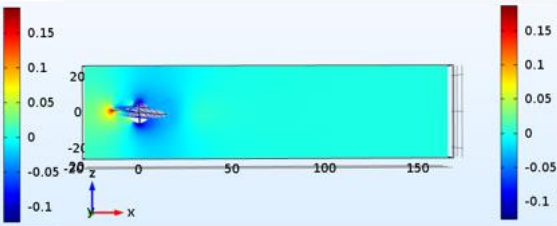

$\alpha=5 \operatorname{deg}$

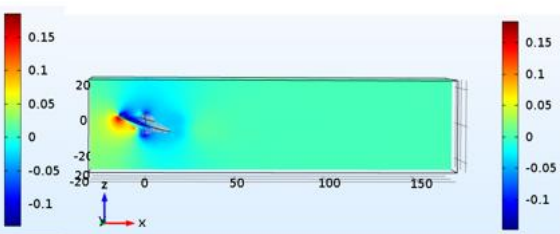

$\alpha=16 \mathrm{deg}$

Figure 9. Pressure profile of Flying Saucer for several angle of attack $(\alpha)$.

The resulted data in the form of Lift and Drag Forces is collected and processed to calculate the Lift and Drag coefficient. The result is shown in Table 1. The coefficient is calculated by divide the force by dynamic pressure and the area of object. 
Table 1. Lift, Drag Forces and Coefficient data.

\begin{tabular}{|c|c|c|c|c|c|}
\hline$\alpha$ [degree] & Lift [N] & Drag [N] & $C_{L}$ & $C_{D}$ & $C_{L} / C_{D}$ \\
\hline 0 & $-1.49 \mathrm{E}-06$ & 8.98E-06 & $-1.37 \mathrm{E}-02$ & 8.30E-02 & $-1.66 \mathrm{E}-01$ \\
\hline 2.5 & $-5.31 E-07$ & $9.70 \mathrm{E}-06$ & $-4.91 E-03$ & 8.97E-02 & $-5.47 \mathrm{E}-02$ \\
\hline 5 & 3.90E-06 & 9.89E-06 & 3.60E-02 & $9.14 \mathrm{E}-02$ & 3.94E-01 \\
\hline 10 & $1.55 \mathrm{E}-05$ & $1.16 \mathrm{E}-05$ & $1.43 \mathrm{E}-01$ & 1.07E-01 & $1.34 \mathrm{E}+00$ \\
\hline 15 & 2.98E-05 & $1.55 \mathrm{E}-05$ & $2.76 \mathrm{E}-01$ & $1.43 \mathrm{E}-01$ & $1.93 \mathrm{E}+00$ \\
\hline 16 & 3.35E-05 & $1.66 \mathrm{E}-05$ & 3.10E-01 & 1.53E-01 & $2.02 \mathrm{E}+00$ \\
\hline
\end{tabular}

The first plot we will discuss is the Aerodynamic coefficients to angle of attack $(\alpha)$ as shown in Figure 10 (a). There are 2 points we will discuss. The first is there is a point where $C_{D}=C_{L}$ at angle of attack $(\alpha)$ around $8 \mathrm{deg}$. The second point is the area beyond angle of attack $(\alpha) 16 \mathrm{deg}$. Since we got divergence result, our plot is discontinued in this area. If we look at the normal plot from a symmetric airfoil such as NACA 0012, in this area the plot should make a convex curve [9]. This area is the maximum $C_{L}$ and then if we increase the angle of attack $(\alpha)$, the stall will occur.

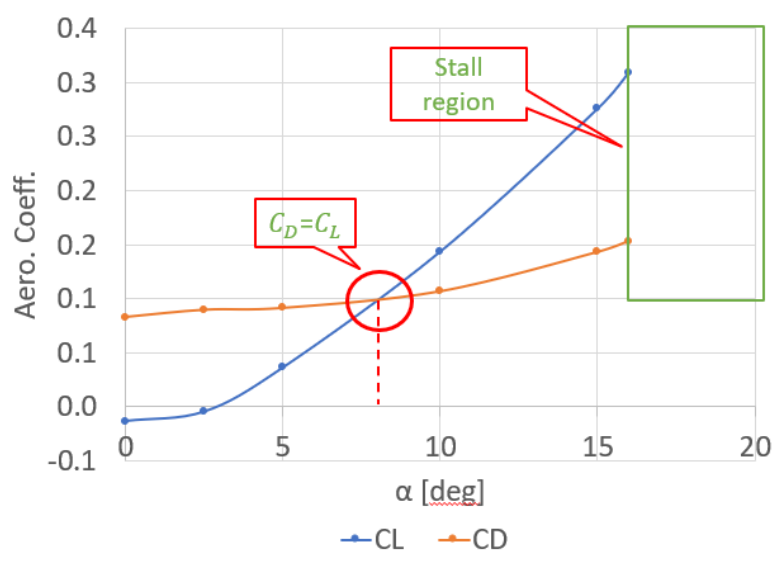

(a)

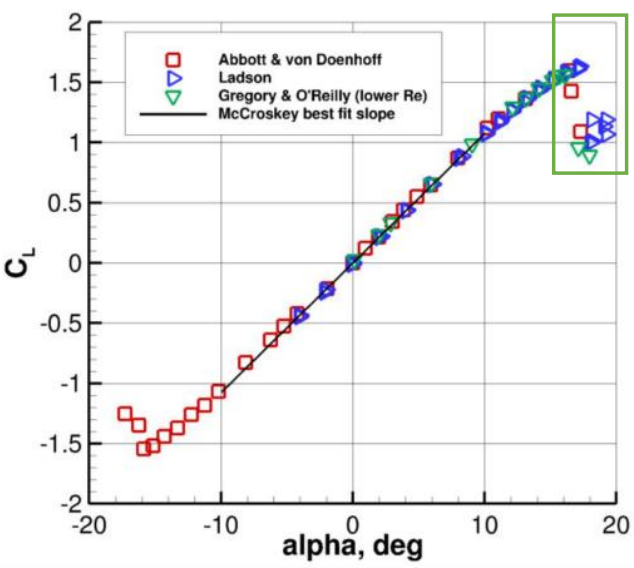

(b)

Figure 10. Aerodynamic Coefficient to Angle of Attack ( $\alpha$ ) Plot: (a) COMSOL result, (b) NACA 0012 data [9]

The second plot we will discuss is the ratio of Aerodynamic coefficient to angle of attack $(\alpha)$ as shown in Figure 11 (a). It is clearly defining that the effective region for Flying Saucer is in the range of angle of attack $(\alpha)$ between 8 to16 deg. Because below that area, the $C_{D}$ generated is more than $C_{L}$. However, in the similar plot resulted from Ring Airfoil Saucer research the effective region is in the range of angle of attack $(\alpha)$ between 11 to15 deg [5]. Thus, our Flying Saucer has larger effective range of angle of attack $(\alpha)$. 


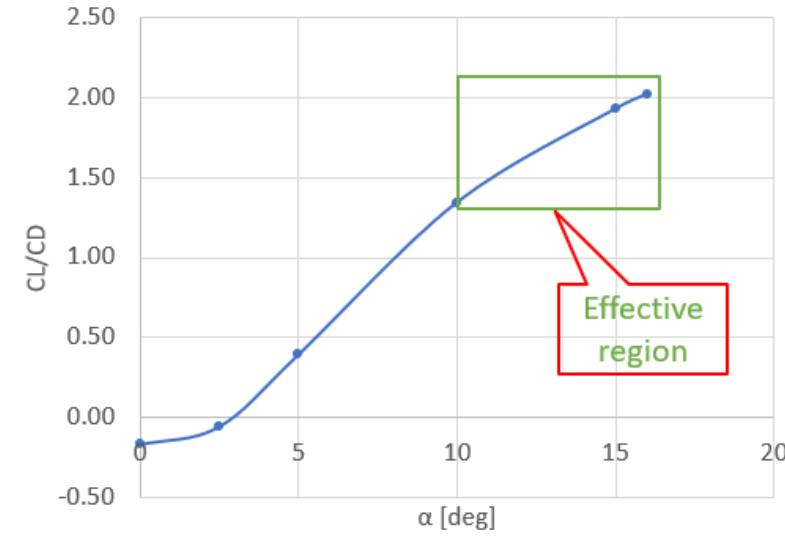

(a)

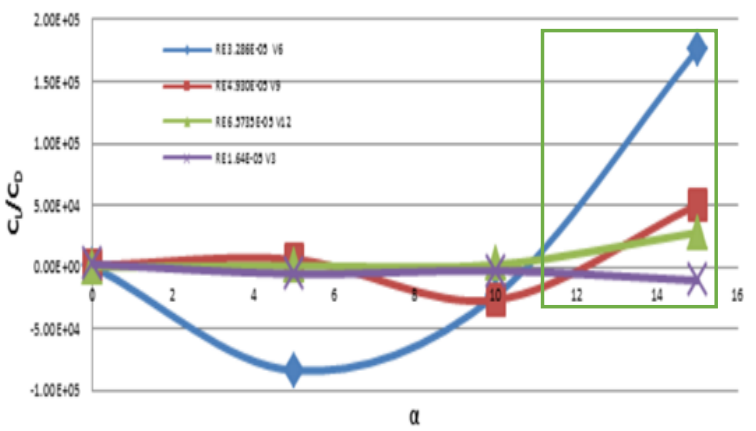

(b)

Figure 11. Ratio of aerodynamic coefficient to angle of attack $(\alpha)$ Plot: (a) COMSOL, (b) Ring airfoil data [5]

The last plot we will discuss is the Drag polar plot as shown in Figure 12 (a). The relation between $C_{L}$ and $C_{D}$ can be established by a second order polynomial $C_{D}=0.4301 C_{L}^{2}+0.0821 C_{L}+0.0871$. The last component of the polynomial we call it $C_{D 0}$ which is the minimum $C_{D}$ that is not function of $C_{L}$.

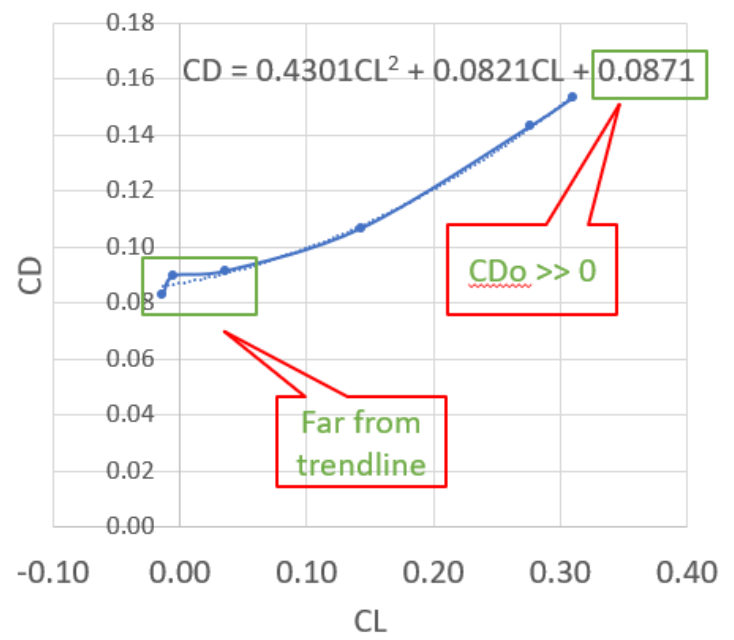

(a)

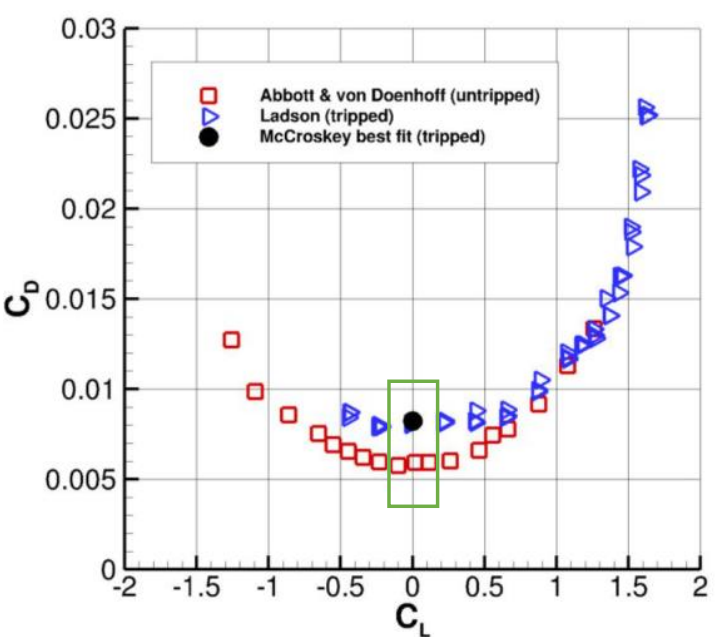

(b)

Figure 12. Drag Polar Plot: (a) COMSOL result, (b) NACA 0012 data [9]

If we compare it with the drag polar plot of NACA 0012, it is more than 10 times [9]. Thus, the minimum Drag coefficient of Flying Saucer is very large. The other highlighted point in this plot is in the area near $C_{L}$ equal to zero where the trendline is not match with the actual data. It is related with the data at angle of attack $(\alpha)$ zero. Thus, we need to explore more in the future work.

\section{Conclusions}

Based on the result and discussion on previous chapter, we can conclude that a symmetric Flying Saucer has aerodynamic characteristics which the maximum angle of attack $(\alpha)$ is 16 degrees, where $C_{L} / C_{D}=2$. The lift became effective above angle of attack $(\alpha)=8 \mathrm{deg}$ where $C_{L} / C_{D}>1$ while the relation between $C_{D}$ and $C_{L}$ was quadratic. The finding of large $C_{D 0}$ was due to the geometric of Flying Saucer. It is recommended that further works is required to explore more detail on area near maximum and 0 angle of attack $(\alpha)$. Validation in wind tunnel experiment is highly recommended. 


\section{References}

[1] I. Grand View Research, “Commercial Drone Market Size To Touch \$2.07 Billion By 2022." https://sites.google.com/site/technologyresearchreport/commercial-drone-market (accessed Jun. 20, 2021).

[2] A. Phillips, “Parrot Introduces the Flying Saucer Drone.” https://dronelife.com/2015/04/01/parrot-flyingsaucer-drone/ (accessed Jun. 20, 2021).

[3] COMSOL, “COMSOL Multiphysics 5.2,” 2021. https://www.comsol.com/ (accessed Jun. 22, 2021).

[4] S. Cortés, D. Güemes, and R. Ávila, “Low Reynolds Number Flow Around a Flying Saucer Micro Air Vehicle," in COMSOL Conference, 2013, pp. 1-7.

[5] A. Aravind-Kumar and S. Periyasamy, "2-D CFD Analysis of Ring Airfoil UAV," Int. J. Sci. Technol. Eng., vol. 3, no. 7, pp. 192-195, 2017.

[6] E. Haque, M. Mashud, and N. Hasan, “Unmanned Aerial Vehicles Construction by Coand ă Effect," Int. Conf. Eng. Res. Innov. Educ. 2017, no. January, 2017.

[7] N. Q. Kelly, Z. Siddiqi, and J. W. Lee, “Computational Fluid Dynamics Analysis and Optimization of the Coanda Unmanned Aerial Vehicle Platform," World Acad. Sci. Eng. Technol. Int. J. Mech. Mechatronics Eng., vol. 14, no. 12, pp. 532-537, 2020.

[8] E. Kuantama and R. Tarca, “Correction of Wind Effect on Quadcopter," 3rd Int. Conf. Sustain. Inf. Eng. Technol. SIET 2018 - Proc., pp. 257-261, 2018, doi: 10.1109/SIET.2018.8693163.

[9] NASA, “2DN00: 2D NACA 0012 Airfoil Validation Case,” 2020. https://turbmodels.larc.nasa.gov/naca0012_val.html (accessed Jun. 22, 2021). 